\title{
Brief Cortical Deactivation Early in Life Has Long-Lasting Effects on Multisensory Behavior
}

\author{
Benjamin A. Rowland, Wan Jiang, and Barry E. Stein \\ Department of Neurobiology and Anatomy, Wake Forest School of Medicine, Medical Center Boulevard, Winston-Salem, North Carolina 27157
}

Detecting and locating environmental events are markedly enhanced by the midbrain's ability to integrate visual and auditory cues. Its capacity for multisensory integration develops in cats 1-4 months after birth but only after acquiring extensive visual-auditory experience. However, briefly deactivating specific regions of association cortex during this period induced long-term disruption of this maturational process, such that even 1 year later animals were unable to integrate visual and auditory cues to enhance their behavioral performance. The data from this animal model reveal a window of sensitivity within which association cortex mediates the encoding of cross-modal experience in the midbrain. Surprisingly, however, 3 years later, and without any additional intervention, the capacity appeared fully developed. This suggests that, although sensitivity degrades with age, the potential for acquiring or modifying multisensory integration capabilities extends well into adulthood.

Key words: colliculus; cross-modal; enhancement; multisensory; orientation; plasticity

\section{Introduction}

Environmental events are often registered by multiple senses, and the brain's ability to integrate the information they provide enhances its responses. That this capability for multisensory integration is present throughout the animal kingdom is testament to its utility in a wide variety of ecological circumstances (Stein and Meredith, 1993; Bolognini et al., 2007; Lakatos et al., 2007; Ross et al., 2007; Zahar et al., 2009). Multisensory integration has been extensively studied in individual superior colliculus (SC) neurons, a midbrain structure involved in the detection and localization of external events as well as in controlling orientation to them. Both the responses of SC neurons and the behaviors they mediate are markedly facilitated by cross-modal cues that are in spatial concordance (multisensory enhancement) (Stein and Rowland, 2011).

The postnatal maturation of these multisensory integration capabilities has been studied most thoroughly in the cat and shown to require experience with the cross-modal cues to be integrated (Wallace and Stein, 1997, 2007; Stein, 2012). Furthermore, their expression in the adult animal depends on the integrity of influences that converge from different unisensory neurons of ipsilateral association cortex (i.e., the anterior ectosylvian sulcus [AES]; and the rostral lateral suprasylvian sulcus [rLS]) (Wallace et al., 1993;

\footnotetext{
Received Sept. 4, 2013; revised March 4, 2014; accepted April 10, 2014.

Author contributions: B.A.R., W.J., and B.E.S. designed research; B.A.R. and W.J. performed research; B.A.R. analyzed data; B.A.R. and B.E.S. wrote the paper.

This work was supported by National Institutes of Health Grants EY016716 and NS036916, the Tab Williams Foundation, and the Wallace Foundation. We thank Huai Jiang for surgical assistance, Nancy London for editorial assistance, and Adam Smith for providing the Elvax used to make the Elvax-muscimol implants.

The authors declare no competing financial interests.

Correspondence should be addressed to Dr. Benjamin A. Rowland, Department of Neurobiology and Anatomy, Wake Forest School of Medicine, Medical Center Boulevard, Winston-Salem, NC 27157. E-mail: browland@wakehealth.edu.

DOI:10.1523/JNEUROSCI.3782-13.2014

Copyright $\odot 2014$ the authors $\quad 0270-6474 / 14 / 347198-05 \$ 15.00 / 0$
}

Jiang et al., 2001; Alvarado et al., 2007, 2009). Depriving the developing animal of relevant cross-modal experience or depriving the adult SC of these cortical influences has similar deleterious effects on the responses of individual SC neurons and the expression of SC-mediated behavior: stimuli from the different sensory modalities elicit appropriate responses, but their crossmodal combinations cannot be integrated to produce response enhancement (Jiang et al., 2002; Wallace et al., 2004; Yu et al., 2010; Xu et al., 2012).

Although these dual maturational requirements have been studied independently, similarities in the consequences of their disruption suggest they are interrelated; specifically, that influences from association cortex play a critical role in encoding the visual-auditory experiences required to craft the neural circuit underlying SC multisensory integration. The objective of the present study was to examine this possibility by evaluating the consequences of temporarily deactivating association cortex during the period in which these SC capabilities normally develop (4-12 weeks postnatal) (Wallace and Stein, 1997). Animals were later studied at maturity (1 year of age) and again later in adulthood (4 years of age). As postulated, even a brief period of cortical deactivation during this period led to long-term behavioral deficits in multisensory integration, but the data also revealed that multisensory integration capabilities could develop much later in life than is normal without further intervention. The results suggest a degree of plasticity in the underlying neural architecture that degrades with age but remains sufficiently robust during adulthood so that, given sufficient time in a normal environment, it can overcome many of the consequences of early insult.

\section{Materials and Methods}

Three domestic cats (Felis domesticus, 1 male and 2 females) from a single litter were used in the behavioral experiments, and an additional $2 \mathrm{fe}$ males were used in a supplementary physiological evaluation. Each ani- 


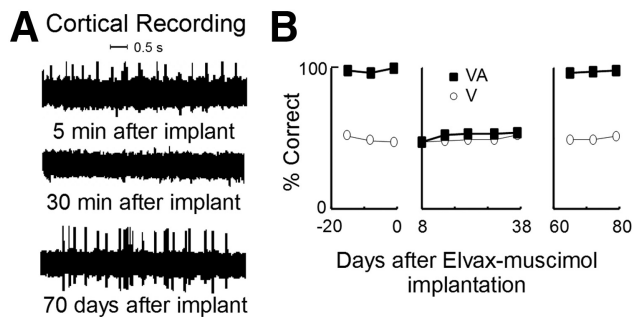

Figure 1. The effect of a brief period of cortical deactivation by muscimol-infused Elvax pledgets. $A$, Extracellular recordings from cortical region AES immediately after implanting the pledget and before its muscimol had an effect on neuronal activity (top), after its effect was evident as the suppression of neuronal activity (middle), and after depletion of the muscimol stores and the return of neuronal activity (bottom). All recordings were taken from the same electrode in an adult control animal. $\boldsymbol{B}$, The accuracy with which the adult localized visual (circles) and spatiotemporally concordant visual-auditory (squares) stimuli before Elvax implantation (left), after implantation and during its effective period (middle), and after muscimol depletion (right). Multisensory and unisensory response accuracies were rendered equivalent (middle) during the period in which the muscimol was effective.

mal was implanted with Elvax polymer pledgets infused with muscimol during postnatal week 3 . The implants were centered over and within the sulcal walls of the AES and rLS unilaterally. The opposite side served as a control in later testing. The muscimol was slowly released over several weeks (Smith et al., 2004), during which time it deactivated the subjacent tissue. The data from these previous experiments and our own confirmatory physiological tests of the implant in the adult cat (Fig. 1) were used to estimate the extent of deactivation in these kittens. The data suggested that a single application of an Elvax-muscimol implant was effective in suppressing cortical activity across all layers in AES and rLS for $\sim 4$ weeks, although the precise effective duration depends on a number of other factors (e.g., changes in brain geometry, such as the depth and extent of sulci) that could not be controlled here. Thus, 4 weeks was taken to be the window of deactivation for a single implant, but the actual extent may have been slightly shorter or longer. Two animals were given a single implant (operational to at least week 7), whereas three received a second implant at this time to extend the estimated deactivation window to at least 11 weeks postnatal. All protocols used were in accordance with the Guide for the care and use of laboratory animals (National Institutes of Health Publication 86-23) and were approved by the Animal Care and Use Committee of Wake Forest Medical School, an Association for the Assessment and Accreditation of Laboratory Animal Careaccredited institution.

Preparation of Elvax-muscimol implants. Elvax-impregnated muscimol implants were prepared using previously described techniques. First, beads of ethylene-vinyl acetate copolymer Elvax 40P (DuPont provided by Smith) were washed for 1 week at room temperature in 95\%-100\% alcohol solution that was changed daily. On the day of preparation, Elvax beads $(0.5 \mathrm{~g})$ were dissolved in dicholoromethane (Sigma-Aldrich) by vortex mixing at room temperature. Muscimol hydrobromide (SigmaAldrich) was dissolved in $100 \mu \mathrm{l}$ of water to yield a $3.75 \mathrm{M}$ muscimol (aq) solution, which was then added to the Elvax suspension and vortex mixed for $10 \mathrm{~min}$. The resulting suspension was then flash-frozen by pouring it onto a glass plate over dry ice. After 1 week of desiccation in a $-75^{\circ} \mathrm{C}$ freezer, the resulting bolus was sectioned on a vibratome at $200 \mu \mathrm{m}$ thickness to produce the Elvax-muscimol pledgets. These pledgets were cut to a desired diameter so that they could be tailored for the size of the cortical region to be deactivated, and soaked in $0.1 \mathrm{~m}$ PBS for $24 \mathrm{~h}$ before implantation.

Implantation of Elvax-muscimol pledgets. Neonates were anesthetized with an initial dose of ketamine hydrochloride $(25 \mathrm{mg} / \mathrm{kg})$ and isoflurane (1-4\%), and anesthesia was maintained with isoflurane (2\%) delivered through a face mask during the surgical procedure. Vital signs were continuously monitored. A unilateral craniotomy was performed and the dura reflected to expose AES and rLS. Elvax-muscimol implants were inserted into the sulci and placed on top of the associated gyri to blanket the region. In 1 animal, a sham implant not containing muscimol was also implanted over the contralateral cortex. The overlying sections of skull were then replaced, implant sites were sutured closed, and a standard regimen of postsurgical analgesics and antibiotics (butorphanol prn, ketoprofen $2 \mathrm{mg} / \mathrm{kg}$, ceftriaxone $20 \mathrm{mg} / \mathrm{kg}$ ) was initiated.

To verify the effectiveness of the Elvax-muscimol implants without compromising the experimental animals, an adult animal previously trained on the localization task (see below) was given an identical surgery and implanted with microwires in AES and rLS for electrophysiological recording. Behavioral tests confirmed that, during the time frame in which the implants were designed to deactivate the subjacent tissue, the animal's multisensory behavioral profile was consistent with animals in which AES and rLS were deactivated cryogenically as in previous studies that have established the role of association cortex in the neural and behavioral manifestations of SC multisensory integration in adults. Electrophysiological recordings from the microwires confirmed that the cortical tissue under the implants was inactive during this period (Fig. 1A). Four weeks after surgery, when the implants lost their effectiveness, the cortex was reactivated and the normal profile of behavioral multisensory enhancement was reinstated (Fig. $1 B$ ).

Behavioral procedure. At 1 year of age, animals were trained and tested on a spatial localization task using previously established methods (Wilkinson et al., 1996; Rowland et al., 2007; Gingras et al., 2009). The researcher conducting the testing did not know which side of the brain contained the implants. Animals were initially trained to stand, facing directly ahead (i.e., at $0^{\circ}$ ), in the center of a 90 -cm-diameter semicircular array of light-emitting diode and speakers placed at $15^{\circ}$ intervals extending $90^{\circ}$ to the left $(-)$ and right $(+)$. The animal was required to approach a briefly $(40 \mathrm{~ms})$ illuminated light-emitting diodes $\left(3.0 \times 10^{-3}\right.$ f.c.) within $3 \mathrm{~s}$ after the stimulus onset to obtain a food reward $(175 \mathrm{mg}$ pellet, Science Diet). To shape this behavior, animals were first trained to approach visual stimuli at $0^{\circ}$, then $\pm 15^{\circ}$, then at any randomly selected location from fixation $\left(0^{\circ}\right)$ to an eccentricity of $60^{\circ}$. Interleaved with these visual-alone trials were catch trials (no stimulus) and auditory trials, in which a speaker located between $-90^{\circ}$ and $90^{\circ}$ was briefly activated, providing a $40 \mathrm{~ms}$ broadband burst of moderately loud $(60 \mathrm{~dB})$ sound. The animal was required to maintain fixation during auditory and catch trials to receive the reward. No cross-modal stimulus combinations were presented during training. Stimuli were pseudo-randomly selected for presentation by custom software and triggered by a foot pedal. Training was complete when the animal responded with $95 \%$ accuracy to each stimulus type measured over 3 consecutive days (i.e., over at least 20 consecutive trials per type).

Once an animal completed this initial training phase, the intensity of the visual stimulus at each location was gradually lowered until $\sim 30 \%$ $50 \%$ correct responding on visual trials was reached (this was designed to maximize sensitivity during testing without compromising the animal's compliance). Auditory stimuli remained at the same level used in training. During a single testing session, visual and auditory stimuli were presented individually at each location ("modality-specific trials") and in cross-modal combinations ("cross-modal trials") at the same location. Table 1 summarizes the stimulus types, numbers, and locations. Stimulus locations were randomly interleaved, with 5-8 trials per condition/ position/d, producing daily estimates of accuracy with measurement error between $\pm 8 \%$ and $\pm 20 \%$. To ensure an equal number of visual and nonvisual (i.e., response-required and no-response-required) trials each day, the number of catch trials was equal to the number of multisensory trials each day (7 or 12). Only correct responses were rewarded during testing.

Initial tests were completed when the animals were 1 year of age, after which they lived in their home cages and were not retested for 3 years ( $\sim 38$ months). Health and behavior were continuously monitored during this period without any observed deviations from normal. At 4 years of age, animals were reacquainted with the task over a period of 2 months and then retested using the same paradigm and criteria. Testing spanned $\sim 36 \mathrm{~d}$ for animals in each age group.

Data analysis. There were 28-34 trial types on each day of testing as shown in Table 1. Responses to each trial type were recorded each day and averaged at each location for each trial type. The principal measure of interest for each trial type was "response accuracy" (localiza- 
Table 1. Mean performance profiles (\% correct localization/\% incorrect localization/\% no-go error) for each animal, each stimulus condition, and each location at 1 and 4 years of age ${ }^{a}$

\begin{tabular}{|c|c|c|c|c|c|c|c|}
\hline & $-45^{\circ}$ & $-30^{\circ}$ & $-15^{\circ}$ & $0^{\circ}$ & $15^{\circ}$ & $30^{\circ}$ & $45^{\circ}$ \\
\hline \multicolumn{8}{|c|}{ Visual alone: 1 year (correct/incorrect/no-go) } \\
\hline 113 & $29 / 41 / 30$ & $34 / 45 / 21$ & $37 / 38 / 24$ & $36 / 45 / 19$ & $27 / 51 / 23$ & $37 / 30 / 33$ & $38 / 34 / 28$ \\
\hline 117 & $44 / 47 / 9.6$ & $55 / 44 / 1.5$ & $38 / 54 / 8.3$ & $51 / 37 / 12$ & $47 / 51 / 1.5$ & $59 / 32 / 9.6$ & $57 / 25 / 17$ \\
\hline 115 & $32 / 66 / 2.7$ & $42 / 53 / 5.6$ & $47 / 47 / 5.9$ & $38 / 52 / 9.5$ & $40 / 50 / 9.8$ & $44 / 44 / 12$ & $38 / 42 / 20$ \\
\hline \multicolumn{8}{|c|}{ Visual-auditory: 1 year (correct/incorrect/no-go) } \\
\hline 113 & $77 / 13 / 11$ & $77 / 8.8 / 14$ & $81 / 9.3 / 9.3$ & $62 / 14 / 24$ & $48 / 20 / 32$ & $48 / 13 / 39$ & $51 / 12 / 37$ \\
\hline 117 & $83 / 12 / 4.6$ & $84 / 11 / 4.7$ & $65 / 33 / 1.5$ & $63 / 27 / 10$ & $60 / 34 / 6.0$ & $65 / 24 / 11$ & $75 / 13 / 12$ \\
\hline 115 & $63 / 30 / 7.1$ & $84 / 12 / 4.4$ & $84 / 15 / 1.5$ & $42 / 48 / 9.9$ & $50 / 29 / 21$ & $45 / 32 / 24$ & $47 / 36 / 17$ \\
\hline \multicolumn{8}{|c|}{ Visual alone: 4 years (correct/incorrect/no-go) } \\
\hline 113 & $17 / 8.3 / 74$ & $47 / 7.8 / 45$ & $48 / 5.8 / 46$ & $42 / 9.0 / 49$ & $23 / 13 / 64$ & $17 / 9.7 / 73$ & $12 / 10 / 77$ \\
\hline 117 & $15 / 14 / 70$ & $35 / 16 / 48$ & $37 / 7.7 / 55$ & $53 / 8.0 / 39$ & $23 / 16 / 62$ & $29 / 9.0 / 62$ & $36 / 8.7 / 55$ \\
\hline 115 & $25 / 4.8 / 70$ & $37 / 10 / 52$ & $24 / 7.5 / 68$ & $42 / 8.2 / 50$ & $29 / 11 / 61$ & $21 / 16 / 63$ & $43 / 12 / 44$ \\
\hline \multicolumn{8}{|c|}{ Visual-auditory: 4 years (correct/incorrect/no-go) } \\
\hline 113 & $54 / 8.6 / 37$ & $77 / 3.9 / 19$ & $65 / 2.0 / 33$ & $67 / 1.9 / 31$ & $54 / 7.1 / 38$ & $39 / 13 / 48$ & $48 / 15 / 37$ \\
\hline 117 & $44 / 31 / 25$ & $72 / 13 / 15$ & 78/4.4/17 & $74 / 4.3 / 22$ & $41 / 11 / 48$ & $60 / 5.5 / 34$ & $54 / 6.0 / 40$ \\
\hline 115 & $56 / 9.7 / 35$ & $56 / 12 / 33$ & $48 / 13 / 39$ & $61 / 5.7 / 33$ & $45 / 15 / 40$ & $51 / 15 / 35$ & $59 / 11 / 30$ \\
\hline
\end{tabular}

${ }^{a}$ Locations ipsilateral to the deactivation are labeled with negative values (e.g., $-45^{\circ}$ ).

tion performance), which was the percentage of responses to the correct location. Also calculated was the incidence of "no-go" errors (where the animal failed to initiate an orientation response to a stimulus) and "incorrect localization" errors (where the animal oriented to the wrong location), in addition to "go" errors on auditory and catch trials, which were exceedingly rare (presumably because of the extensive training).

The mean and variance (across days) of these variables were calculated for each location and testing block. To quantify multisensory enhancement, the proportionate multisensory index (MSI) was calculated $(100 \times$ multisensory response accuracy - unisensory response accuracy/unisensory response accuracy). A "predicted" multisensory response magnitude was generated for each location in space contralateral to the implants using the unisensory accuracy and the MSI calculated at the corresponding location in ipsilateral (i.e., control) space. Response patterns were quantified at each location and examined for evidence of changes in response bias in the multisensory conditions. Mann-Whitney $U$ tests were used to establish significant differences between performance categories. Interhemispheric conditions were compared by comparing performance across the two hemifields, ignoring data collected with target locations at fixation $\left(0^{\circ}\right)$. Data across locations and animals were pooled to generate summary statistics.

Physiological recordings. SC recordings were conducted to provide supplementary physiological evidence. Two cohorts of animals were used: a cohort of 2 female cats given the muscimol-infused Elvax implants at 4 and 8 weeks of age and studied at 1 year of age, and the cohort of animals used in the behavioral study upon its completion (now 4 years of age). Electrophysiological procedures were identical to those described in detail previously (Yu et al., 2010; Xu et al., 2012). Briefly, animals were first instrumented with a head-holding recording well (McHaffie and Stein, 1983) then, after full recovery, were used in recording experiments. For all experiments, animals were anesthetized with ketamine hydrochloride $(20 \mathrm{mg} / \mathrm{kg}$, i.m. initial dose, and maintained with $5-10 \mathrm{mg} / \mathrm{kg} / \mathrm{h})$, and acepromazine maleate $(0.1 \mathrm{mg} / \mathrm{kg}, \mathrm{i} . \mathrm{m}$.), artificially respired, and paralyzed with pancuronium bromide $(0.1 \mathrm{mg} / \mathrm{kg}$, i.v., initial dose and maintained with $0.05 \mathrm{mg} / \mathrm{kg} / \mathrm{h}$ ) and $5 \%$ dextrose solution. Glass-coated tungsten electrodes (impedance: $1-3 \mathrm{M} \Omega$ at $1 \mathrm{kHz}$ ) recorded single neuron responses to visual (moving bars of light), auditory (broadband noise bursts), and somatosensory (cutaneous stimulation) stimuli that were presented individually and in combination. Multisensory integration was evaluated in each neuron based on the statistical enhancement of its responses to spatiotemporally concordant cross-modal stimuli as in the past (Meredith and Stein, 1983). Stimulus presentations consisted of pseudo-randomly interleaved modality-specific and cross-modal stimuli (20 trials/condition).
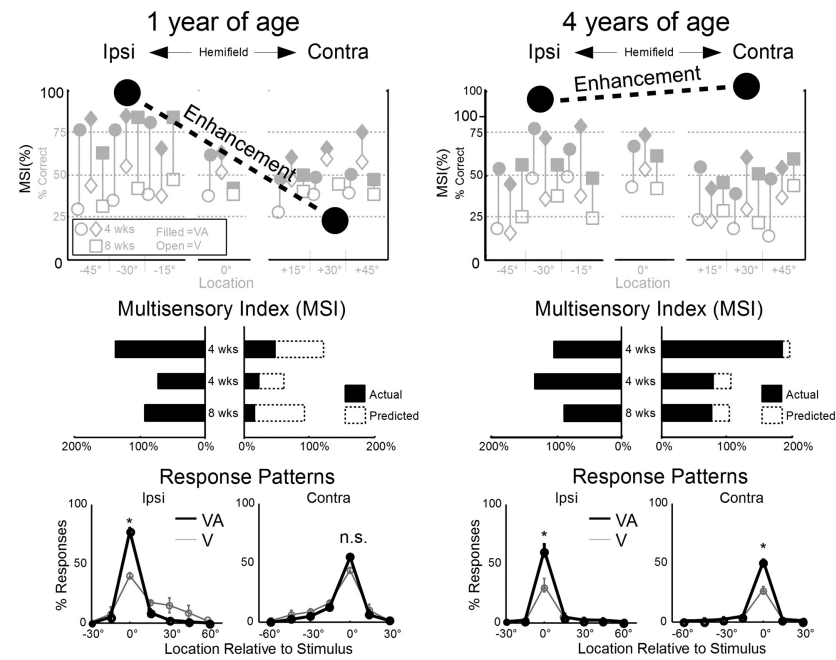

Figure 2. Following brief early life deactivation of the left association cortex (AES and rLS), multisensory enhancement was absent in the right hemifield at 1 year of age but present at 4 years of age. Top, Large black circles and dotted line represent the average enhancement (MSI) recorded in the hemifield ipsilateral (ipsi) and contralateral (contra) to the previously deactivated cortex at 1 year (left) and 4 years (right) of age. In the background are the percentage of correct responses to visual (open icons) and visual-auditory (filled icons) stimuli for each animal (circles, diamonds represent animals given 4 weeks of deactivation; squares represent animals given 8 weeks of deactivation) at each location. Middle, Average MSI scores for each animal at all ipsilateral (left) and contralateral (right) locations. Dashed bars on the right indicate the predicted MSI. There is significant degradation of performance to contralateral stimuli. Bottom, The proportion of stimulus-elicited responses recorded to each location, relative to the actual stimulus location, averaged in ipsilateral (left) and contralateral (right) space. Relative to the unisensory response probabilities (thin gray line), multisensory response probabilities (solid black line) were typically elevated at the correct response location $\left(0^{\circ}\right)$ but not other (erroneous) locations. The exception to this rule was found in contralateral space at 1 year of age, where the multisensory and unisensory responses were equivalent. ${ }^{*} p<0.01 ;$ n.S., Not significant, $p>$ 0.01 .

\section{Results}

All animals were able to localize the visual stimulus in contralateral and ipsilateral space equally well at 1 year of age after intensities were calibrated (ipsilateral, $40 \pm 8 \%$ vs contralateral, $43 \pm 10 \%)$. Nevertheless, a consistent and statistically significant defect in multisensory enhancement was evident at every contralateral location tested in every animal (Fig. 2, left). Thus, 
A

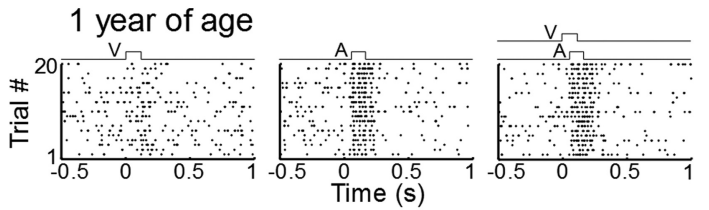

4 years of age

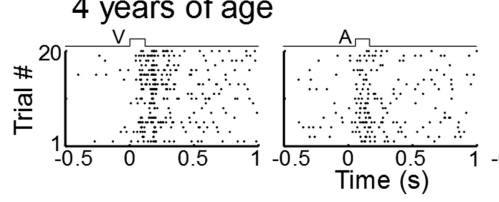

B

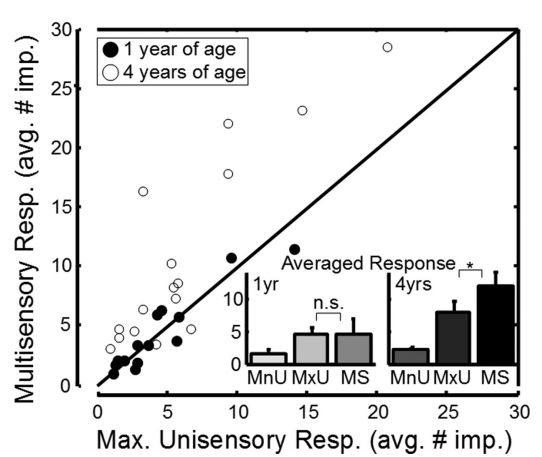

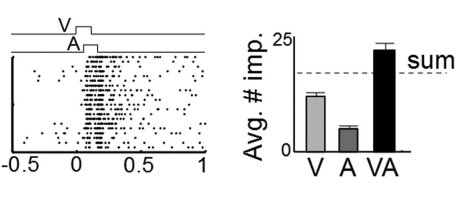

C

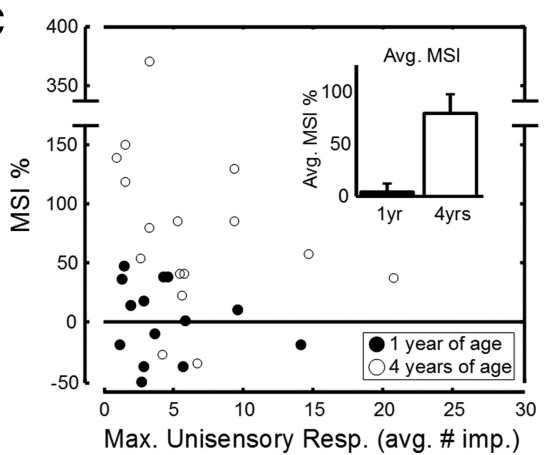

Figure 3. Changes in multisensory enhancement capabilities were also observed in SC physiology. A, Recordings from exemplar multisensory SC neurons ipsilateral to early cortical deactivation at 1 year of age (top) and 4 years of age (bottom). Responses to a cross-modal (visual-auditory) stimulus were no more robust than to the most effective component stimulus at 1 year but were significantly enhanced at 4 years. Bar graphs on the right represent the magnitude of each response (average no. of stimuluselicited impulses). Error bars indicate SEM. $\boldsymbol{B}$, The patterns of enhancement observed in the individual neurons in $\boldsymbol{A}$ were reflected in populations of ipsilateral $S C$ multisensory neurons recorded at 1 year of age (filled circles, $n=14$ ) and 4 years of age (open circles, $n=16$ ) in animals given these treatments. Plotted is the multisensory response magnitude (average no. of impulses) versus the maximum of the component unisensory response magnitudes for each neuron. Inset, Averaged response magnitudes across each population for three categories: the minimum unisensory component response (MnU), the maximum unisensory response (MxU), and the multisensory response (MS). C, The relationship between MSI and the maximum unisensory response for the neurons recorded in the two age groups. Error bars indicate SEM. Inset, Average MSI across these two cohorts. As in the behavioral tests, multisensory integration capabilities that were compromised at 1 year of age had developed at 4 years of age.

localization of the cross-modal stimulus was significantly $(p<$ $0.01)$ less accurate in contralateral than ipsilateral space (ipsilateral, $78 \pm 18 \%$ vs contralateral, $55 \pm 16 \%)$. Furthermore, although marginally significant (at $p \sim=0.03$ ), the multisensory enhancement (MSI) evoked by contralateral cross-modal stimuli $(27 \pm 15 \%)$ was $72 \%$ less than predicted based on responses to the same stimuli in ipsilateral space ( $97 \pm 32 \%)$. Response patterns indicated significant elevation of correct responses in the multisensory conditions in ipsilateral space without corresponding increases in incorrect responses to nearby locations; however, no such enhancement was observed in contralateral space. Each animal's data trends were consistent with the population trend, although the animal given 8 weeks of cortical deactivation showed somewhat greater multisensory defects in contralateral space (MSI 81\% smaller than predicted) than the animals given 4 weeks of deactivation (MSI 67\% smaller than predicted). In sum, all animals were substantially compromised in their ability to integrate visual and auditory stimuli in the contralateral hemifield even 9-10 months after neonatal cortical deactivation was terminated.

However, at 4 years of age, animals no longer showed any statistically significant $(p>0.05)$ interhemispheric differences in unisensory or multisensory performance (Fig. 2 , right). Nor were there significant interhemispheric differences in MSI $(p=0.86)$, which averaged $109 \pm 54 \%$ in ipsilateral space versus $118 \pm 78 \%$ in contralateral space. When these values were compared with those at 1 year of age, it was apparent that, whereas MSI in ipsilateral space had not changed significantly $(p=0.60)$, the average MSI in contralateral space had markedly $(p<0.0003)$ improved (by $337 \%)$. Although there were some idiosyncratic variations in performance at different contralateral locations, they were not significantly deviant from the population trend. The response patterns at 4 years of age were equivalent on both sides of space and showed the same multisensory enhancement observed in ipsilateral space at 1 year of age. The number of incorrect localization responses was lower at 4 years of age; and although the cause of this change was not obvious, it was independent of (i.e., uncorrelated with) the relationship between the unisensory and multisensory responses.

Supplementary physiological examination (Fig. 3) of SC multisensory neurons yielded results consistent with the behavioral observations at 1 and 4 years of age. At 1 year of age, the responses to concordant cross-modal stimuli were almost never (1 of 14) significantly enhanced in neurons of the SC ipsilateral to the cortex that had been deactivated (mean MSI = $3.5 \%)$. However, in the 4-year-old animals, physiological enhancement was common (11 of 16) and significantly more efficacious (mean MSI $=78.6 \%$ ). The results from individual neurons (Fig. $3 A$ ) were consistent with those from the populations of neurons recorded (Fig. $3 B, C$ ).

\section{Discussion}

The present results reveal that deactivating association cortex for a brief period during the first few postnatal months of life has a surprisingly long-term disruptive effect on the ability of the SC to develop the ability to integrate cross-modal stimuli and thereby enhance the salience of their initiating events. This deficit was manifested behaviorally as an absence of cross-modal facilitation in a simple localization task. The results are consistent with the hypothesis that association cortex must be functional for the brain to use cross-modal experience to craft the midbrain circuit underlying multisensory enhancement in orientation and localization tasks (Wallace and Stein, 1997; Jiang et al., 2002, 2006, 2007; Yu et al., 2013). Although there is little direct information regarding the specific biophysical mechanisms involved in this maturational process, one prominent hypothesis is that a basic Hebbian mechanism is at work, either via projections from cortex to SC or via recurrent loops. In such a mechanism, correlated activity between sensory-specific neuronal ensembles within visual and auditory regions of AES/rLS and common target neurons in the ipsilateral SC selectively potentiate convergent afferents (Cuppini et al., 2011, 2012). This strengthening occurs in ensembles whose sensitivities match the properties of the cross-modal events they encounter and, as a consequence, the circuit becomes adapted to the likely environment in which it will be used.

Particularly notable was that the profound impairment of multisensory integration capabilities noted in early adulthood 
was not permanent. When the animals were tested several years later, all showed statistically normal multisensory enhancement capabilities. It is, of course, impossible to know whether their general multisensory processing capabilities had actually achieved the "normal" state. Nonetheless, it was striking to note that the multisensory enhancement deficits were ameliorated in the absence of rehabilitative intervention (Foxe et al., 2013), albeit requiring far more time and experience than is otherwise required. Apparently, there is a sensitive period during early life in which influences from association cortex facilitate the brain's ability to use experience with cues from different senses to build a circuit for their synergistic use. This, in turn, enhances the impact of events providing such cues and sensitizes the circuit to the environment in which it will be used. Although this flexibility degrades substantially thereafter, it may allow refinements in this process to continue throughout adulthood. These data also have implication for rehabilitative strategies aimed at ameliorating the multisensory deficits that have been noted in a variety of developmental disorders (e.g., Hairston et al., 2005; Williams et al., 2010; Stevenson et al., 2014).

\section{References}

Alvarado JC, Stanford TR, Vaughan JW, Stein BE (2007) Cortex mediates multisensory but not unisensory integration in superior colliculus. J Neurosci 27:12775-12786. CrossRef Medline

Alvarado JC, Stanford TR, Rowland BA, Vaughan JW, Stein BE (2009) Multisensory integration in the superior colliculus requires synergy among corticocollicular inputs. J Neurosci 29:6580-6592. CrossRef Medline

Bolognini N, Leo F, Passamonti C, Stein BE, Làdavas E (2007) Multisensorymediated auditory localization. Perception 36:1477-1485. CrossRef Medline

Cuppini C, Stein BE, Rowland BA, Magosso E, Ursino M (2011) A computational study of multisensory maturation in the superior colliculus (SC). Exp Brain Res 213:341-349. CrossRef Medline

Cuppini C, Magosso E, Rowland B, Stein B, Ursino M (2012) Hebbian mechanisms help explain development of multisensory integration in the superior colliculus: a neural network model. Biol Cybern 106:691-713. CrossRef Medline

Foxe JJ, Molholm S, Del Bene VA, Frey HP, Russo NN, Blanco D, SaintAmour D, Ross LA (2013) Severe multisensory speech integration deficits in high-functioning school-aged children with autism spectrum disorder (ASD) and their resolution during early adolescence. Cereb Cortex. Advance online publication. Retrieved Aug. 28, 2013. doi: 10.1093/ cercor/bht213. CrossRef Medline

Gingras G, Rowland BA, Stein BE (2009) The differing impact of multisensory and unisensory integration on behavior. J Neurosci 29:4897-4902. CrossRef Medline

Hairston WD, Burdette JH, Flowers DL, Wood FB, Wallace MT (2005) Altered temporal profile of visual-auditory multisensory interactions in dyslexia. Exp Brain Res 166:474-480. CrossRef Medline

Jiang W, Wallace MT, Jiang H, Vaughan JW, Stein BE (2001) Two cortical areas mediate multisensory integration in superior colliculus neurons. J Neurophysiol 85:506-522. Medline

Jiang W, Jiang H, Stein BE (2002) Two corticotectal areas facilitate multisensory orientation behavior. J Cogn Neurosci 14:1240-1255. CrossRef Medline

Jiang W, Jiang H, Stein BE (2006) Neonatal cortical ablation disrupts multisensory development in superior colliculus. J Neurophysiol 95:13801396. CrossRef Medline
Jiang W, Jiang H, Rowland BA, Stein BE (2007) Multisensory orientation behavior is disrupted by neonatal cortical ablation. J Neurophysiol 97: 557-562. CrossRef Medline

Lakatos P, Chen CM, O'Connell MN, Mills A, Schroeder CE (2007) Neuronal oscillations and multisensory interaction in primary auditory cortex. Neuron 53:279-292. CrossRef Medline

McHaffie JG, Stein BE (1983) A chronic headholder minimizing facial obstructions. Brain Res Bull 10:859-860. Medline

Meredith MA, Stein BE (1983) Interactions among converging sensory inputs in the superior colliculus. Science 221:389-391. CrossRef Medline

Ross LA, Saint-Amour D, Leavitt VM, Javitt DC, Foxe JJ (2007) Do you see what I am saying? Exploring visual enhancement of speech comprehension in noisy environments. Cereb Cortex 17:1147-1153. CrossRef Medline

Rowland B, Stanford T, Stein B (2007) A Bayesian model unifies multisensory spatial localization with the physiological properties of the superior colliculus. Exp Brain Res 180:153-161. CrossRef Medline

Smith AL, Parsons CH, Lanyon RG, Bizley JK, Akerman CJ, Baker GE, Dempster AC, Thompson ID, King AJ (2004) An investigation of the role of auditory cortex in sound localization using muscimol-releasing Elvax. Eur J Neurosci 19:3059-3072. CrossRef Medline

Stein BE, ed (2012) The new handbook of multisensory processing. Cambridge, MA: MIT.

Stein BE, Meredith MA (1993) The merging of the senses. Cambridge, MA: MIT.

Stein BE, Rowland BA (2011) Organization and plasticity in multisensory integration: early and late experience affects its governing principles. Prog Brain Res 191:145-163. CrossRef Medline

Stevenson RA, Siemann JK, Schneider BC, Eberly HE, Woynaroski TG, Camarata SM, Wallace MT (2014) Multisensory temporal integration in autism spectrum disorders. J Neurosci 34:691-697. CrossRef Medline

Wallace MT, Stein BE (1997) Development of multisensory neurons and multisensory integration in cat superior colliculus. J Neurosci 17:2429 2444. Medline

Wallace MT, Stein BE (2007) Early experience determines how the senses will interact. J Neurophysiol 97:921-926. CrossRef Medline

Wallace MT, Meredith MA, Stein BE (1993) Converging influences from visual, auditory, and somatosensory cortices onto output neurons of the superior colliculus. J Neurophysiol 69:1797-1809. Medline

Wallace MT, Ramachandran R, Stein BE (2004) A revised view of sensory cortical parcellation. Proc Natl Acad Sci U S A 101:2167-2172. CrossRef Medline

Wilkinson LK, Meredith MA, Stein BE (1996) The role of anterior ectosylvian cortex in cross-modality orientation and approach behavior. Exp Brain Res 112:1-10. CrossRef Medline

Williams LE, Light GA, Braff DL, Ramachandran VS (2010) Reduced multisensory integration in patients with schizophrenia on a target detection task. Neuropsychologia 48:3128-3136. CrossRef Medline

Xu J, Yu L, Rowland BA, Stanford TR, Stein BE (2012) Incorporating crossmodal statistics in the development and maintenance of multisensory integration. J Neurosci 32:2287-2298. CrossRef Medline

Yu L, Rowland BA, Stein BE (2010) Initiating the development of multisensory integration by manipulating sensory experience. J Neurosci 30: 4904-4913. CrossRef Medline

Yu L, Xu J, Rowland BA, Stein BE (2013) Development of cortical influences on superior colliculus multisensory neurons: effects of dark-rearing. Eur J Neurosci 37:1594-1601. CrossRef Medline

Zahar Y, Reches A, Gutfreund Y (2009) Multisensory enhancement in the optic tectum of the barn owl: spike count and spike timing. J Neurophysiol 101:2380-2394. CrossRef Medline 